\title{
POLR2A deletion with TP53 opens a window of opportunity for therapy
}

Inactivation of the guardian of the genome, the tumour suppressor protein $\mathrm{p} 53$, is common in most human tumours. Despite tremendous efforts, no effective therapy aimed at restoring p53 activity has been successfully translated into the clinic. Now, Xiongbin $\mathrm{Lu}$ and colleagues highlight that co-depletion of TP53 and one of its neighbouring genes, $P O L R 2 A$, renders cancer cells highly vulnerable to POLR2A inhibition, providing a new treatment strategy for tumours containing such genomic changes.

Copy-number losses targeting tumour suppressors often involve neighbouring genes that do not contribute to cancer development per se, but nevertheless generate a vulnerability that is exploitable for therapeutic development.

"The POLR2A gene is essential for cell survival, and is almost always co-deleted with TP53 in many human cancers, including colorectal, breast, ovarian, kidney, liver, and pancreatic cancer," explains Lu. Analysis of The Cancer Genome Atlas database has revealed that deletion of POLR2A occurs in virtually all the human colorectal cancers (CRC) with hemizygous TP53 deletion.

"Despite improvements in medical research and surgical provision, there has been little change in mortality rates in CRC in the past decades, with limited treatment options, especially for CRC possessing TP53 deletion," says Lu. Notably, POLR2A, which encodes the largest subunit of RNA polymerase II, is druggable and can be specifically inhibited with a-amanitin.

Hemizygous loss of POLR2A results in decreased abundance of RNA polymerase II in CRC cells, suggesting that these cells could be more vulnerable to $\alpha$-amanitin inhibition than unaltered normal cells. Importantly, the researchers have demonstrated

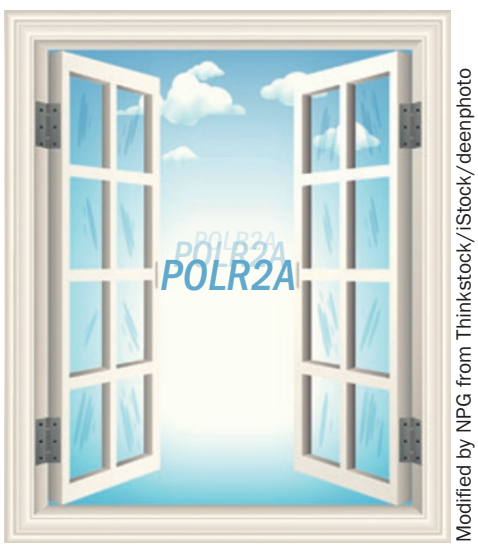

that suppression of POLR2A with a-amanitin (or small interfering RNAs) selectively inhibits the proliferation, survival and tumorigenic potential of CRC cells with hemizygous TP53 loss, in a p53-independent manner.

Despite the strong inhibitory effect of $\alpha$-amanitin, the liver toxicity associated with this agent has presented a major challenge to its clinical development. However, such toxicity can be overcome by the conjugation of $a$-amanitin with monoclonal antibodies against EpCAM, a cellsurface marker that is enriched in adenocarcinomas, including CRC. Of note, the use of antibody-drug conjugates of $\alpha$-amanitin also provides targeting specificity.

"We are planning to expand our preclinical studies with $\alpha$-amanitin antibody conjugates from CRC to other types of human cancers with hemizygous loss of TP53 and POLR2A, and search for other POLR2A-targeted small chemical compounds," concludes Lu. The principle of collateral vulnerability to POLR2A inhibition is opening a window of opportunity for new, and hopefully efficient, cancer therapy.

Alessia Errico

Original article Liu, Y. et al. TP53 loss creates therapeutic vulnerability in colorectal cancer. Nature 520,697-701 (2015) 